\title{
Need for a structured policy for discharging high-risk preterm neonatal intensive care unit graduate in the coronavirus disease pandemic era
}

\author{
Purbasha Mishra ${ }^{1}$, Pankaj Kumar Mohanty ${ }^{2}$, Tapas Kumar Som ${ }^{2}$, Mohammad Zakiulla ${ }^{1}$, Tanushree Sahoo ${ }^{3}$ \\ From ${ }^{1}$ DM Resident, ${ }^{2}$ Associate Professor, ${ }^{3}$ Assistant Professor, Department of Neonatology, All India Institute of Medical Sciences, Bhubaneswar, \\ Odisha, India
}

Dear Sir,

$\mathrm{I}$ $\mathrm{n}$ the era of the coronavirus disease 2019 (COVID-19) pandemic, where the world is struggling to contain the spread of infection and prevent mortality, preterm neonates are becoming very much vulnerable. Although we witness asymptomatic COVID-positive neonates mostly at the same time, getting symptomatic neonates may not be a rarity in the future. The neonatal age group, especially the preterm babies due to their immunological immaturity, is much more vulnerable to develop sepsis and pneumonia. A recent meta-analysis in antenatal mothers with COVID positive revealed that the risk of iatrogenic preterm birth and cesarean delivery had increased considerably [1]. We should be prepared for the unprecedented footfall of symptomatic neonates in the future. Although most born neonates born to COVID-positive mothers are asymptomatic, as the transplacental transmission is a rarity, the horizontal transfer of infection is very much possible if precautions are not taken adequately $[2,3]$. In the present scenario, we should be extremely cautious in discharging preterm infants, especially those with chronic lung disease.

There were 64 cases of COVID real-time polymerase chain reaction (RT-PCR)-positive mothers whose babies were born at our hospital. Out of them, five babies were COVID positive. We had the policy to do COVID RT-PCR in those babies who are symptomatic. A neonate born to COVID-19-positive mother is symptomatic if the neonate developed fever, respiratory distress [in the form of tachypnea (more than $60 / \mathrm{min}$ ), chest wall retraction (subcostal or intercostal)], apnea, feeding difficulty, or loose motion [4].

Among all five positive cases, three were symptomatic. There were two preterm babies (36 weeks and 33 weeks) and one term babies. Two babies develop transient tachypnea of the newborn, and all three babies had perinatal asphyxia without hypoxicischemic encephalopathy. One moderate preterm baby (33 weeks) developed bronchopulmonary dysplasia (BPD).

One moderate preterm was born to COVID-positive mother by vaginal delivery due to preterm premature rupture of membranes.

\section{Access this article online}

Received -18 October 2020

Initial Review - 26 November 2020

Accepted - 13 February 2021

DOI: 10.32677/IJCH.2021.v08.i02.010
The baby did not cry after birth required bag and mask ventilation (BMV) for $15 \mathrm{~min}$; the baby was subsequently ventilated in neonatal intensive care unit (NICU). Birth weight was $890 \mathrm{~g}$. The baby was intubated and ventilated with assisted controlled mode, received surfactant for respiratory distress syndrome (RDS), and was extubated in $75 \mathrm{~h}$ of life. The chest X-ray (CXR) before the administration showed signs of RDS (low volume lung and reticulogranular opacity). Total parenteral nutrition was started on day 1. COVID RT-PCR was positive for the baby. Minimal enteral feeds were started on day 3 . On day 6 , the baby had increased Fio2 requirement and developed intercostal retraction.

On examination, a systolic murmur was heard in the left upper sternal border. Echocardiography revealed significant hemodynamic patent ductus arteriosus (PDA). The baby received intravenous paracetamol for 3 days. Repeat ECHO revealed PDA closed. The baby had to be again intubated as the Fio2 requirement increased, and the baby developed shock and metabolic acidosis. The sepsis screen was positive; blood culture grew pseudomonas. The baby received appropriate antibiotics according to unit protocol. Cerebrospinal fluid (CSF) study was negative for meningitis. The baby was extubated to continuous positive airway pressure (CPAP) (PEEP 5/ Fio2 30\%) on day 8 of life, which was continued till day 13 . The baby was on heated, humidified high-flow delivered by nasal cannulae support (fio2 $21 \%$ ) till day 80 of life. The baby had BPD, required low flow oxygen up to day 80 , and then was in room air. In between, the baby was managed for cholestasis, acute kidney injury, hypoglycemia, and thrombocytopenia. The baby was discharged successfully on day 85 at a corrected gestational age (GA) 45 weeks with a birth weight of $1530 \mathrm{~g}$. On follow-up, the baby was doing well, gaining weight, and millstones in an age-appropriate manner.

Recently, we witnessed few cases more preterm with BPD, which were discharged and again readmitted for respiratory issues within a month interval. The readmission happened just before the arrival of the COVID-19 pandemic in the subcontinent, this threw several questions regarding our discharge policy for preterm babies; especially, in the COVID pandemic era.

Correspondence to: Pankaj Kumar Mohanty, Department of Neonatology, All India Institute of Medical Sciences, Bhubaneswar, Odisha, India. E-mail: neonat_pankaj@aiimsbhubaneswar.edu.in

(C) 2021 Creative Commons Attribution-NonCommercial 4.0 International License (CC BY-NC-ND 4.0). 
The first case was about a baby, born at 29 weeks, $895 \mathrm{~g}$, by emergency lower segment cesarean section (LSCS). The baby did not cry after birth, received, BMV for 1 min; baby developed tachypnea for which received early CPAP. CXR showed signs of RDS. The baby received an early rescue surfactant. Echocardiography and ultrasound cranium were normal. The baby was discharged on day 62 but readmitted on day 66 for lethargy, apnea. The baby had to be intubated. Late onset of sepsis was presumed. The sepsis screen was negative. CXR showed bilateral opacities in the lung field. Blood and CSF cultures were sterile. Urine cytomegalovirus was negative, and fungal blood culture was sterile. The baby developed acute RDS (ARDS) and required high-frequency ventilation. Tracheal aspirate grew influenza B, for which oseltamivir was given. The baby developed shock, disseminated intravascular coagulation, and succumbed on day 82.

The second baby was born at 28 weeks of GA, vaginal delivery because of premature contraction. After initial steps, the baby developed tachypnea so connected to CPAP. CXR showed signs of RDS, intubation, surfactant replacement, and extubation was done. The baby required increased $\mathrm{FiO}_{2}$ on CPAP, so the baby was intubated again at $72 \mathrm{~h}$ of life patient-triggered ventilation. The sepsis screen was negative, and the baby had tachycardia, for which echocardiography was done, which revealed hemodynamic significant PDA. Oral ibuprofen was given; PDA closed. The baby was CPAP dependent (FiO2 21\%, PEEP 5), hemodynamically stable, packed red blood cells transfused for low hematocrit, gaining weight, on day 40 removed from CPAP, kangaroo mother care (KMC) being done. The baby was discharged on day 45 and was again readmitted on day 55 for tachypnea and chest wall retraction. The baby had signs of upper respiratory tract infection; CXR revealed signs of viral pneumonia. CPAP was connected to the baby the baby improved and was discharged on day 65 .

The third baby was moderate preterm ( 32 weeks), born through LSCS, $1095 \mathrm{~g}$. The baby had a weak cry at birth, then required BMV for $30 \mathrm{~s}$, was shifted to NICU and connected to CPAP $\left(\mathrm{FiO}_{2}\right.$ $25 \%$, PEEP 5). The baby could not be removed and developed chronic lung disease. On $84 \mathrm{~h}$, the baby required increased $\mathrm{FiO}_{2}$ to maintain saturation. The CXR showed bilateral lung opacities. The baby was intubated and ventilated, sepsis screen was positive. The baby received antibiotics pending blood culture. Blood and CSF cultures were sterile. The baby was extubated to CPAP on day 7 , removed from CPAP on day $40^{\text {th }}$ of life, and discharged on day 50 . The baby was again readmitted on day 72 for apnea and decreased feeding. On arrival, the baby was lethargic and apneic. The baby was immediately connected to CPAP. CXR showed bilateral lung opacities (new lesion as compared to the previous X-ray). Arterial blood gas revealed metabolic acidosis; the sepsis screen was negative, blood and CSF cultures were sterile. Tracheal aspirate grew Mycoplasma hominis. The baby was treated with azithromycin for 10 days, was removed from CPAP on day 80 , discharged on day 85 , and on follow-up baby was doing well.

The above three cases alerted us to be extremely cautious before discharging any preterm baby with respiratory complications.
Whether infants being discharged in-home oxygen or in-room air are going to be particularly crucial for policy-making. We saw in the above three babies with chronic lung disease returned with community-acquired viral infection/pneumonia, out of which one baby succumbed due to ARDS. During the COVID pandemic; especially, if the mother is symptomatic, treated, and got discharged after being well, specific precautions should be taken for such babies. We might be witnessing more cases of premature babies who got discharged home, and the mother might be COVID positive. It is essential to follow the steps meticulously while discharging preterm infants from the hospital in the COVID pandemic era. We also need to focus on other viruses causing respiratory infection along with COVID-19. There is no increase in the incidence of BPD in preterm but if the pandemic continues and rising incidence of prematurity will affect BPD incidence.

The post-discharge problems should be adequately addressed. These babies are prone to readmission due to respiratory infection, feeding issues, morbidities related to any system, and failure to thrive. They should be put into a structured follow-up program, initiated.

\section{HOSPITAL POLICY FOR DISCHARGE AND FOLLOW-UP}

A policy should be made involving the stakeholders. The parents or secondary caregivers, doctors, and nursing personal should be involved in policy-making. Parental education, their access to online platforms such as WhatsApp/Telegram, or any other application should be considered. If they come from a remote place, then nearby health establishments should be enquired and communicated to them to ease health assessment in case of emergency. Any "Swasthya app," if available, parents should be trained to make good use of it.

\section{FEEDING MOTHERS OWN MILKAND MAINTAINING GOOD NUTRITION}

The baby should be directly breastfed or fed with expressed breast milk. Breastfeeding should be encouraged in every situation, mothers and infant dyads should be cared for together, and skin-to-skin contact must be ensured. The WHO recommends exclusive breastfeeding irrespective of COVID status. If the mother is COVID positive, breastfeeding must be allowed with some precautions like maintain hand hygiene and using a face mask [5]. Failure to thrive should be addressed appropriately. All medications, including anti-gastroesophageal reflux if required, should be added. Proper nutrition and preventing sepsis are going to be crucial for management. Regular follow-up in the hospital may not be feasible in pandemic times, so parents should consult the nearest health establishment for growth assessment. If not possible, then at least or home weight checking and feeding behavior should be checked. An online platform can be used to follow up where parents can send details of their baby or a video of the child regarding any issue/concern. 


\section{MAINTAINING HYGIENE AND ASEPSIS}

Proper hand hygiene, using the face mask, social distancing in the home should be followed. The mother should comply with hand hygiene, respiratory hygiene. Those having any signs of upper respiratory tract infection should be barred from coming near the baby. Neonatologist or treating pediatrician should use optimum precautions/personal protective equipment while managing the baby. COVID appropriate behavior should be followed by parents, and health-care personal should teach and convince them to adopt it.

\section{IMMUNIZATION}

All routine immunizations should be given at birth irrespective of the COVID status of the baby. Pneumococcal vaccine at 6 weeks should be given [6]. Social distancing, mask, and sanitization should be strictly followed and recommended COVID-19-related norms should be strictly observed in the immunization session. A uniform dose of seasonal flu vaccine $(15 \mathrm{mg}$ or $0.5 \mathrm{ml})$ can be given after 6 months. A baby with BPD should be administered the pneumococcal vaccine. Palivizumab (monoclonal antibody) may be considered if available, which is produced by recombinant DNA technology used to prevent respiratory infection caused by a respiratory syncytial virus.

\section{FOLLOW-UP AND DISCHARGE PREPAREDNESS}

The Parents should be counseled in detail about follow-up care, picking danger signs. The screening for retinopathy of prematurity (ROP) must have been done, and proper redressal of follow-up should be in place. Babies with no ROP or Zone III Stage 1 or 2 may differ from regular follow-up protocol. Hearing screening and congenital heart disease screening critical congenital heart disease must be complete before discharge. Proper nutrition and maintaining asepsis are essential for optimum growth and development. Patents are motivated to continue KMC at home. Developmental supportive care and early stimulation must be initiated when babies are admitted to NICU, and parents should be involved actively in the program so that they can continue the task at ease at home for the optimal neurodevelopmental outcome. In case, the baby being discharged with home oxygen (few babies with severe BPD who are unable to wean from oxygen); extra precaution should be taken to prevent infection. Hypoxia and hyperoxia should be avoided, and oxygen requirements should be tailored according to the need to maintain adequate saturation. They should have a pulse oximeter for checking saturation. They should be given training on how to check oxygen saturation. If the saturation drops below $<90 \%$, they should contact the health-care facility. Ideally, they should buy a weighing machine so that the weight of the baby can be checked at regular intervals. Preferably, they should be given a teleconsultancy number to directly video call to the health facility.

\section{READMISSION POLICY}

The readmission of preterm babies should clearly be defined, and parents should get optimum information before discharging their infants from an intensive care unit. They should be advised to maintain respiratory hygiene. The readmission happened in our case for viral infection mostly. In case, a baby comes to the parent hospital from the community and admission if warranted, the COVID test (preferably RT-PCR) should be performed, and the baby should be managed accordingly [7].

Proper follow-up of the babies is going to be a challenge in COVID times. Acute care and long-term follow-up threw a great challenge to us; public awareness programs for the public should be undertaken. Health establishments should make necessary and structured policies so that vulnerable groups can get optimum health benefits.

\section{REFERENCES}

1. Khalil A, Kalafat E, Benlioglu C, O'Brien P, Morris E, Draycott T, et al. SARS-CoV-2 infection in pregnancy: A systematic review and metaanalysis of clinical features and pregnancy outcomes. EClinicalMedicine 2020;25:100446.

2. Egloff C, Vauloup-Fellous C, Picone O, Mandelbrot L, Roques P. Evidence, and possible mechanisms of rare maternal-fetal transmission of SARSCoV-2. J Clin Virol 2020;128:104447.

3. Chen H, Guo J, Wang C, Luo F, Yu X, Zhang W, et al. Clinical characteristics and intrauterine vertical transmission potential of COVID-19 infection in nine pregnant women: A retrospective review of medical records. Lancet 2020;395:809-15.

4. Dumpa V, Kamity R, Vinci AN, Noyola E, Noor A. Neonatal coronavirus 2019 (COVID-19) infection: A case report and review of literature. Cureus 2020;12:e8165.

5. Lubbe W, Botha E, Niela-Vilen H, Reimers P. Breastfeeding during the COVID-19 pandemic a literature review for clinical practice. Int Breastfeed J 2020;15:82.

6. Kasi SG, Dhir S, Verma S, Pemde HK, Balasubramanian S, Agarwalla S, et al. Immunization during the COVID-19 pandemic: Recommendations from Indian academy of pediatrics advisory committee on vaccines and immunization practices. Indian Pediatr 2020;57:1147-52.

7. World Health Organization. Laboratory testing for 2019 Novel Coronavirus (2019-nCoV) in Suspected Human Cases: Interim Guidance; 2020. Available from: https://www.who.int/publications-detail/laboratory-testing-for-2019novel-coronavirus-in-suspected-human-cases-20200117. [Last accessed on 2020 Mar 05].

Funding: None; Conflicts of Interest: None Stated.

How to cite this article: Mishra P, Mohanty PK, Som TK, Zakiulla M, Sahoo T. Need for a structured policy for discharging high-risk preterm neonatal intensive care unit graduate in the coronavirus disease pandemic era. Indian J Child Health. 2021; 8(2):106-108. 